EDWARD NEUMAN

\title{
OPTIMAL BOUNDS FOR CERTAIN BIVARIATE MEANS
}

\begin{abstract}
New bivariate means, introduced and investigated in [1], play a central role in this work. The lower and upper bounds for those means are obtained. Bounding quantities are the oneparameter means derived from the harmonic and contraharmonic means by forming convex combinations of the variables of these two means.
\end{abstract}

Key words: Bivariate means, one-parameter means, optimal bounds, inequalities.

2010 Mathematical Subject Classification: 26E60, $26 D 0 \%$.

\section{$\S 1$. Introduction}

Recently several researchers have obtained optimal bounds for some bivariate means such as logarithmic mean, two Seiffert means, NeumanSándor mean, Neuman means, to mention few. The optimal bounds are the other bivariate means which are simpler than those for which the optimal bounds are sought. For more details the interested reader is referred to $[1-7]$ and the references therein.

This paper deals with optimal lower and upper bounds for the family of bivariate means introduced in [1] and is organized as follows. In Section 2 we give definition and some basic properties of the generic one-parameter family of means. Definitions of bivariate means used in this paper are given in Section 3. In particular, we include there formulas for the four new means investigated in [1]. Optimal bounds for these means are established in Section 4. The bounding quantities are the one-parameter generalizations of either the harmonic or the contraharmonic means.

(C) Edward Neuman, 2014 


\section{$\S 2$. Definition and basic properties of the one-parameter family of means}

For the reader's convenience we recall definition and basic properties of the one-parameter family of bivariate means. Following [3] we define two nonnegative numbers $w_{1}$ and $w_{2}$ :

$$
w_{1}=\frac{1+p}{2}, \quad w_{2}=\frac{1-p}{2},
$$

where the parameter $p$ satisfies $0 \leq|p| \leq 1$. Clearly $w_{1}+w_{2}=1$. In what follows $(a, b)$ will stand for the pair of unequal positive numbers. We associate with $(a, b)$ another pair of positive numbers $(x, y)$, where

$$
x=w_{1} a+w_{2} b, \quad y=w_{1} b+w_{2} a .
$$

Thus $x$ and $y$ are the convex combinations of $a$ and $b$. One can easily verify that (for $0<p \leq 1$ ) $a<x<y<b$ if $a<b$ or $b<y<x<a$ if $b<a$.

For the sake of presentation let $\mathcal{N}$ stand for a generic bivariate symmetric mean. We define a one-parameter mean $\mathcal{N}_{p}(a, b) \equiv \mathcal{N}_{p}$ as follows

$$
\mathcal{N}_{p}(a, b)=\mathcal{N}(x, y) .
$$

In the sequel we will call mean $\mathcal{N}_{p}$ the p-mean or the p-mean generated by $\mathcal{N}$.

We recall now some elementary properties of the p-means. Using (2.3), (2.1), and (2.2) we see that

$$
\mathcal{N}_{-p}(a, b)=\mathcal{N}(y, x)=\mathcal{N}(x, y)=\mathcal{N}_{p}(a, b) .
$$

Thus the function $p \rightarrow \mathcal{N}_{p}$ is an even function. Without a loss of generality we may assume that $0 \leq p \leq 1$. It follows from (2.1) and (2.2) that

$$
\mathcal{N}_{0}=A, \quad \mathcal{N}_{1}=\mathcal{N}
$$

Moreover, the function $p \rightarrow \mathcal{N}_{p}$ is strictly decreasing if $\mathcal{N}<A$, i. e.,

$$
\mathcal{N}_{1} \leq \mathcal{N}_{p} \leq N_{0}
$$

or is strictly increasing if $\mathcal{N}>A$, i. e.,

$$
\mathcal{N}_{0} \leq \mathcal{N}_{p} \leq \mathcal{N}_{1}
$$




\section{$\S 3$. Bivariate means used in this paper}

In this section we give definitions and formulas of certain bivariate means used in the next section of this paper.

First we define a number $v$ as follows

$$
v=\frac{a-b}{a+b}
$$

Clearly $0<|v|<1$.

The arithmetic, harmonic and contraharmonic means of $a$ and $b$, denoted respectively by, $A, H$ and $C$, are defined in usual way

$$
A=\frac{a+b}{2}, \quad H=\frac{2 a b}{a+b} \quad \text { and } \quad C=\frac{a^{2}+b^{2}}{a+b} .
$$

One can easily verify that

$$
H=A\left(1-v^{2}\right) \quad \text { and } \quad C=A\left(1+v^{2}\right) .
$$

The p-means generated by $H$ and $C$ are denoted, respectively, by $H_{p}$ and $C_{p}$. It is an elementary task to show that

$$
H_{p}=A\left(1-(p v)^{2}\right) \quad \text { and } \quad C_{p}=A\left(1+(p v)^{2}\right) .
$$

We recall now formulas for the four means which play a central role in this paper. They have been introduced and studied in [1]:

$$
\begin{gathered}
N_{A G}=\frac{1}{2} A\left(1+\left(1-v^{2}\right) \frac{\tanh ^{-1} v}{v}\right), \\
N_{G A}=\frac{1}{2} A\left(\sqrt{1-v^{2}}+\frac{\sin ^{-1} v}{v}\right) \\
N_{Q A}=\frac{1}{2} A\left(\sqrt{1+v^{2}}+\frac{\sinh ^{-1} v}{v}\right) \\
N_{A Q}=\frac{1}{2} A\left(1+\left(1+v^{2}\right) \frac{\tan ^{-1} v}{v}\right)
\end{gathered}
$$

where the symbols $G$ and $Q$ stand for the geometric and the root-square means of $a$ and $b$, respectively. Recall that

$$
G=\sqrt{a b} \quad \text { and } \quad Q=\sqrt{\frac{a^{2}+b^{2}}{2}} .
$$


The four means defined in (3.3)-(3.6) are special cases of the SchwabBorchardt mean $S B$ which is defined as follows

$$
S B(a, b) \equiv S B= \begin{cases}\frac{\sqrt{b^{2}-a^{2}}}{\cos ^{-1}(a / b)} & \text { if } a<b, \\ \frac{\sqrt{a^{2}-b^{2}}}{\cosh -1(a / b)} & \text { if } b<a\end{cases}
$$

(see, e.g., [9], [10]). This mean has been studied extensively in [11], [12], and in [13]. It is well known that the mean $S B$ is strict, nonsymmetric and homogeneous of degree one in its variables. The Schwab-Borchardt mean is the iterative mean, i. e.,

$$
S B=\lim _{n \rightarrow \infty} a_{n}=\lim _{n \rightarrow \infty} b_{n}
$$

where

$$
a_{0}=a, \quad b_{0}=b, \quad a_{n+1}=\frac{a_{n}+b_{n}}{2}, \quad b_{n+1}=\sqrt{a_{n+1} b_{n}}
$$

$(n=0,1, \ldots)$.

The four means under discussion are defined in terms of $S B, A, G$ and $Q$ as follows: $N_{A G}=S B(A, G), N_{G A}=S B(G, A), N_{Q A}=S B(Q, A)$ and $N_{A Q}=S B(A, Q)$.

All the means mentioned above are comparable. It is known that (see [5, Theorem 4.2])

$$
H<G<N_{A G}<N_{G A}<A<N_{Q A}<N_{A Q}<Q<C .
$$

As in [1] we call means given in (3.3) - (3.6) the four new means.

\section{$\S 4$. Optimal bounds for the four new means}

The goal of this section is to obtain optimal bounds for the four new means defined in the previous section with the bounding quantities being either the p-mean generated the harmonic mean or the p-mean generated by the contraharmonic mean.

In the proofs presented in this section we will utilize the following result (see, e.g., [14]). 
Theorem A. Let the functions $f$ and $g$ be continuous on $[c, d]$, differentiable on $(c, d)$ and such that $g^{\prime}(t) \neq 0$ on $(c, d)$. If $f^{\prime}(t) / g^{\prime}(t)$ is (strictly) increasing (decreasing) on $(c, d)$, then the functions

$$
\frac{f(t)-f(d)}{g(t)-g(d)} \text { and } \frac{f(t)-f(c)}{g(t)-g(c)}
$$

are also (strictly) increasing (decreasing) on $(c, d)$.

In what follows we will assume that the numbers $p$ and $q$ are belong to the unit interval $[0,1]$. We are in a position to prove the following result.

Theorem 1. The following two-sided inequality

$$
H_{p}<N_{A G}<H_{q}
$$

is valid provided

$$
p \geq \sqrt{\frac{1}{2}}=0.707106 \ldots \quad \text { and } \quad q \leq \sqrt{\frac{1}{3}}=0.577350 \ldots .
$$

Proof. Making use of (3.3) and (3.2) we see that the two-sided inequality (4.1) inequality can be written as follows

$$
q^{2}<\frac{1}{2 v^{2}}\left[1-\left(1-v^{2}\right) \frac{\tanh ^{-1} v}{v}\right]<p^{2} .
$$

Letting $v=\tanh t(t>0)$ we obtain

$$
q^{2}<\phi_{1}(t)<p^{2}
$$

where

$$
\phi_{1}(t)=\frac{f(t)}{g(t)}:=\frac{\sinh t \cosh ^{2} t-t \cosh t}{2 \sinh ^{3} t} .
$$

Differentiation yields

$$
\frac{f^{\prime}(t)}{g^{\prime}(t)}=\frac{3 \sinh 2 t-2 t}{6 \sinh 2 t}=\frac{1}{6}\left(3-\frac{2 t}{\sinh 2 t}\right):=h_{1}(t) .
$$

Since the function $2 t / \sinh 2 t$ is strictly decreasing on the interval $(0, \infty)$ we conclude that the function $h_{1}(t)$ is strictly increasing on the same domain. Application of Theorem A leads to the conclusion that the function 
$\phi_{1}(t)$ is also strictly increasing on the interval $(0, \infty)$. One can easily verify that $\phi_{1}\left(0^{+}\right)=1 / 3$ and $\phi_{1}\left(\infty^{-}\right)=1 / 2$. This completes the proof of (4.1) with the domains of validity for $p$ and $q$ as stated in (4.2).

In the next theorem we give optimal bounds for the mean $N_{G A}$ in terms of the p-mean generated by the harmonic mean.

Theorem 2. The inequalities

$$
H_{p}<N_{G A}<H_{q}
$$

are satisfied if

$$
p \geq \sqrt{1-\frac{\pi}{4}}=0.463252 \ldots \quad \text { and } \quad q \leq \sqrt{\frac{1}{6}}=0.408248 \ldots
$$

Proof. First we shall write the double inequality (4.3) in the equivalent form. Making use of (3.4) together with the application of the first part of (3.2), followed by a substitution $v=\sin t(0<t<\pi / 2)$, yields after a little algebra,

$$
q^{2}<\phi_{2}(t)<p^{2}
$$

where

$$
\phi_{2}(t)=\frac{f(t)}{g(t)}:=\frac{2 \sin t-t-\sin t \cos t}{2 \sin ^{3} t} .
$$

Differentiation gives

$$
\frac{f^{\prime}(t)}{g^{\prime}(t)}=\frac{1}{6} \sec ^{2}\left(\frac{t}{2}\right):=h_{2}(t) .
$$

Clearly the function $h_{2}(t)$ is strictly increasing on the interval $(0, \pi / 2)$. We utilize Theorem A again to conclude that the function $\phi_{2}$ is also strictly increasing on the same interval. It is easy to verify that

$$
\phi_{2}\left(0^{+}\right)=\frac{1}{6} \quad \text { and } \quad \phi_{2}\left(\frac{\pi}{2}\right)=1-\frac{\pi}{4} .
$$

This in conjunction with (4.5) yields the bounds for $p$ and $q$ as stated in (4.4).

In the following theorem we shall establish optimal bounds $C_{p}$ and $C_{q}$ for the mean $N_{Q A}$. 
Theorem 3. If $p=0 \quad$ and $\quad q \geq \sqrt{\frac{1}{6}}=0.408248 \ldots$,

then

$$
C_{p}<N_{Q A}<C_{q} \text {. }
$$

Proof. We follow the lines of the proofs of two theorems already established in this section. Making use of the second formula of (3.2) and (3.5) we can write inequality (4.7) in the equivalent form as

$$
p^{2}<\frac{1}{v^{2}}\left[\frac{1}{2}\left(\sqrt{1+v^{2}}+\frac{\sinh ^{-1} v}{v}\right)-1\right]<q^{2} .
$$

With the substitution $v=\sinh t(t>0)$ the last double inequality becomes

$$
p^{2}<\phi_{3}(t)<q^{2}
$$

where

$$
\phi_{3}(t)=\frac{f(t)}{g(t)}:=\frac{\sinh t \cosh t+t-2 \sinh t}{2 \sinh ^{3} t} .
$$

Differentiating $f(t)$ and $g(t)$ we obtain, upon simplifications,

$$
\frac{f^{\prime}(t)}{g^{\prime}(t)}=\frac{1}{6 \cosh ^{2}(t / 2)}:=h_{3}(t) .
$$

Clearly function $h_{3}(t)$ is strictly decreasing on the interval $(0, \infty)$. This in conjunction with Theorem A leads to the conclusion that the function $\phi_{3}(t)$ is strictly decreasing on the the positive semi-axis. Utilizing L'Hôpital rule we obtain $\phi_{3}\left(0^{+}\right)=1 / 6$ and $\phi_{3}\left(\infty^{-}\right)=0$. Combining this with (4.8) yields (4.6). The proof is complete.

We close this section with the following.

Theorem 4. The two-sided inequality

$$
C_{p}<N_{A Q}<C_{q}
$$

holds true if

$$
p \leq \frac{\sqrt{\pi-2}}{2}=0.534226 \ldots \quad \text { and } \quad q \geq \sqrt{\frac{1}{3}}=0.577350 \ldots
$$


Proof. Making use of the second formula of (3.2) and (3.6) one can rewrite inequality (4.9) as follows

$$
p^{2}<\frac{1}{2 v^{2}}\left[\left(1+v^{2}\right) \frac{\tan ^{-1} v}{v}-1\right]<q^{2}
$$

With $v=\tan t(0<t<\pi / 4)$ the last two-sided inequality becomes

$$
p^{2}<\phi_{4}(t)<q^{2},
$$

where

$$
\phi_{4}(t)=\frac{f(t)}{g(t)}:=\frac{t \cos t-\sin t \cos ^{2} t}{2 \sin ^{3} t} .
$$

This yields

$$
\frac{f^{\prime}(t)}{g^{\prime}(t)}=\frac{1}{6}\left(3-\frac{2 t}{\sin 2 t}\right):=h_{4}(t) .
$$

Taking into account that the function $2 t / \sin 2 t$ is strictly increasing on the interval $(0, \pi / 4)$ we conclude that the function $h_{4}(t)$ is strictly decreasing on the same interval. This in turn, in view of Theorem A, implies that the function $\phi_{4}(t)$ is strictly decreasing. Simple calculations yield

$$
\phi_{4}\left(0^{+}\right)=\frac{1}{3} \quad \text { and } \quad \phi_{4}(\pi / 4)=\frac{\pi-2}{4} .
$$

This in conjunction with (4.11) yield the bounds (4.10).

\section{References}

[1] Neuman E. On a new bivariate mean. Aequationes Math., in press.

[2] He Z.-Y., Chu Y.-M., Wang M.-K. Optimal bounds for Neuman means in terms of harmonic and contraharmonic means. J. Appl. Math., to appear.

[3] Neuman E. A one-parameter family of bivariate means. J. Math. Inequal., 2013, vol. 7, no. 3, pp. 399-412.

[4] Neuman E. Sharp inequalities involving Neuman-Sándor and logarithmic means. J. Math. Inequal., 2013, vol. 7, no. 3, pp. 413-419.

[5] Neuman E. On generalized Seiffert means. Aequationes Math., 2014, vol. 87, no. 3, pp. 325-335. 
[6] Neuman E. On some means derived from the Schwab-Borchrdt mean. J. Math. Inequal., 2014, vol. 8, no. 1, pp. 171-183.

[7] Neuman E. Inequalities involving certain bivariate means II. J. Inequal. Spec. Funct., 2013, vol. 4, no. 4, pp. 12-20.

[8] Neuman E. Inequalities involving certain bivariate means. Bull. Internat. Math. Virtual Inst., 2013, vol. 3, pp. 49-57.

[9] Borwein J. M., Borwein P. B. Pi and the AGM: A Study in Analytic Number Theory and Computational Complexity. John Wiley and Sons, New York, 1987.

[10] Carlson B. C.Algorithms involving arithmetic and geometric means. Amer. Math. Monthly, 1971, vol. 78, pp. 496-505.

[11] Neuman E., Sándor J.On the Schwab-Borchardt mean. Math. Pannon., 2003, vol. 14, no. 2, pp. 253-266.

[12] Neuman E., Sándor J.On the Schwab-Borchardt mean II. Math. Pannon., 2006, vol. 17, no. 1, pp. 49-59.

[13] Neuman E. Inequalities for the Schwab-Borchardt mean and their applications. J. Math. Inequal., 2011, vol. 5, pp. 601-609.

[14] Alzer H., Qiu S.-L. Monotonicity theorems and inequalities for complete elliptic integrals. J. Comput. Appl. Math., 2004, vol. 172, pp. 289-312.

The work is received on December 12, 2013.

Mathematical Research Institute,

144 Hawthorn Hollow, Carbondale, IL 62903, USA.

E-mail: edneuman76@gmail.com 